\title{
A Monte Carlo Template based analysis for Air-Cherenkov Arrays
}

\author{
R.D. Parsons ${ }^{\mathrm{a}, *}$, J.A. Hinton ${ }^{\mathrm{b}}$ \\ ${ }^{a}$ Max-Planck-Institut für Kernphysik, P.O. Box 103980, D 69029, Heidelberg, Germany \\ ${ }^{b}$ Department of Physics and Astronomy, The University of Leicester, University Road, Leicester, LE1 7RH, United \\ Kingdom
}

\begin{abstract}
We present a high-performance event reconstruction algorithm: an Image Pixel-wise fit for Atmospheric Cherenkov Telescopes (ImPACT). The reconstruction algorithm is based around the likelihood fitting of camera pixel amplitudes to an expected image template. A maximum likelihood fit is performed to find the best-fit shower parameters. A related reconstruction algorithm has already been shown to provide significant improvements over traditional reconstruction for both the CAT and H.E.S.S. experiments. We demonstrate a significant improvement to the template generation step of the procedure, by the use of a full Monte Carlo air shower simulation in combination with a ray-tracing optics simulation to more accurately model the expected camera images. This reconstruction step is combined with an MVA-based background rejection.

Examples are shown of the performance of the ImPACT analysis on both simulated and measured (from a strong VHE source) gamma-ray data from the H.E.S.S. array, demonstrating an improvement in sensitivity of more than a factor two in observation time over traditional image moments-fitting methods, with comparable performance to previous likelihood fitting analyses. ImPACT is a particularly promising approach for future large arrays such as the Cherenkov Telescope Array (CTA) due to its improved highenergy performance and suitability for arrays of mixed telescope types.
\end{abstract}

Keywords: Gamma-ray astronomy; IACT; Analysis technique; Cherenkov technique

\section{Introduction}

Ground-based gamma-ray astronomy exploits the air shower produced by the interaction of a primary gamma ray in the Earth's atmosphere. For gamma-rays of approximately $10^{11}-10^{14} \mathrm{eV}$ (VHE) few air-shower particles reach ground level. However, sufficiently high-energy secondary particles will emit Cherenkov radiation, resulting in illumination of a $\sim 10^{5} \mathrm{~m}^{2}$ patch of ground for a few nanoseconds. The Imaging Atmospheric Cherenkov Technique (IACT) is based on the observation of this emission by a number of large reflecting telescopes, placed within the light pool with a typical spacing of $\sim 100 \mathrm{~m}$. The Cherenkov light is focussed onto ultra-fast, but typically rather coarsely pixelated, cameras, resulting in roughly elliptical images of the shower emission in multiple telescopes. H.E.S.S. [1] is an array of four such $100 \mathrm{~m}^{2}$ reflecting telescopes, at $1800 \mathrm{~m}$ altitude in the Khomas Highlands of Namibia, each

\footnotetext{
* Corresponding author

Email Address: Daniel.Parsons@mpi-hd.mpg.de Tel: +496221 516137
}

with a 960 pixel camera viewing a $5^{\circ}$ diameter patch of sky.

The process of reconstruction/estimation of the properties of the primary photon (direction and energy), and the rejection of events likely to belong to the background of hadronic showers, makes use of image information from each telescope. Traditionally this event reconstruction is performed using the Hillas parameters of the camera images 2], derived after an image cleaning step (described in [3]). The Hillas parameters are the moments of the camera image which, given the approximately elliptical nature of typical camera images, already capture much of the available image information. In the most commonly used stereoscopic reconstruction method the major axes of images are calculated in a common camera reference frame and the intersection points of all axes found. A weighted average (based on image amplitude and the angle between the axes) is then taken of all crossing points to provide an estimate of the arrival direction of the primary gamma-ray. A similar procedure involving the intersections of the directions between the image centroid and the optical axis is then performed in a common plane perpendicular 
to the pointing direction, to determine the shower impact point on the ground.

Although relatively good angular resolution ( $\sim 1^{\circ}$ with H.E.S.S.) can be reached with such a reconstruction procedure 3], significant additional information can be extracted from the recorded images in a typical event, resulting in improved performance. Additionally a simultaneous fit procedure between all telescopes can help to ensure a consistent result is found between all telescopes, rather than the independent analysis used in Hillas reconstruction. An image template fitting procedure was pioneered for the CAT telescope [4] 5] and re-implemented and improved upon for H.E.S.S. [6]. These methods begin with the creation of a semi-analytical model of air-shower development and IACT response, and use this model to generate the expected shower image for a given set of shower parameters (primary energy and direction, and also impact distance of the shower from the telescope). The template library can then be compared to the images recorded in a given event, and, by means of a multi-dimensional fit procedure, the best-fit shower parameters determined. An alternative method of shower fitting has also been developed for use with H.E.S.S. data, fitting the pixel intensities of the camera image with the expectation from a simpler analytical 3 dimensional gaussian air shower model (3D model) [7, 8].

One of the major problems with the model and 3D model analyses is the difficulty of describing the air shower behaviour at high energies $(>10 \mathrm{TeV})$. Above $10 \mathrm{TeV}$ a large number of particles reach ground level, resulting in large fluctuations which are difficult to reproduce with the aforementioned approaches. This difficultly in reproducing energetic air showers results in poor event reconstruction above $10 \mathrm{TeV}$, typically leading to a rapid drop in effective collection area. With these approaches it is also difficult to account for instrumental effects, such as the optical point spread function of the telescopes or the limited readout window of the camera, so approximations of these effects must be made. Inevitably, making these kind of assumptions will limit the quality of the model fit, reducing the accuracy of the analysis.

One way to solve these problems with high energy events is to instead produce templates by the use of detailed Monte Carlo (MC) based air shower simulations. Such an approach requires no analytical approximations to be made within the air shower simulation, and therefore takes better into account the large fluctuations arising from particles reaching ground level. Additionally, a more accurate optics and electronics simulation can be performed on the simulated Cherenkov photons, resulting a more realistic representation of the tele- scope behaviour. The MC approach is a robust and general image template generation method, not requiring any adaptation to the model for different telescope types, instead existing Monte Carlo telescope configurations can be adapted to produce templates.

Below we present an attempt to improve the accuracy of the template generation, by the use of a more accurate Monte Carlo simulation based air shower model, combined with a ray-tracing telescope simulation and demonstrate the resultant improvement in the performance of the analysis.

\section{Template Generation}

In contrast to the semi-analytical model of shower development used in [5] \& [6] to generate image templates, we use instead the CORSIKA/sim_telarray chain [9, 10] to perform a Monte Carlo air shower simulation, followed by ray-tracing of the telescope optics and simulation of the instrument electronics. The use of this very detailed and computing intensive simulation chain has several major benefits over the, faster, semianalytical approach.

Firstly, the CORSIKA air shower simulation is a well proven code, which has been shown to be consistent with the results of multiple experiments, in combination with the sim_telarray telescope simulation it has been shown to accurately reproduce results from H.E.S.S.. The use of a complete air shower simulation also allows the effects of the geomagnetic field on the air shower to be incorporated, which proves difficult in analytical models. Finally the simulation of the electronics chain using sim_telarray allows instrumental effects, such as trigger biases and readout window length (very important in the case of H.E.S.S.) to be naturally accounted for.

There are, however, some drawbacks to the MC approach, firstly, in order to fill the same parameter space of templates, a significantly larger computing time is required, due to the CPU intensive nature of MC simulations. Also, with such a random approach the edges of the parameters space are naturally sparsely populated, making the fitting of unusual events more difficult. This issue of phase space coverage was most apparent close to the array trigger threshold, where an extremely large number of event simulations were required in order to reproduce the relatively few upfluctuations that trigger the array.

Full MC simulation of a number of air showers (300,000 - 600 depending on the primary gammaray energy) were performed for a given event parameter set and traced through the simulation of the optics. Any photons reaching the focal plane in 

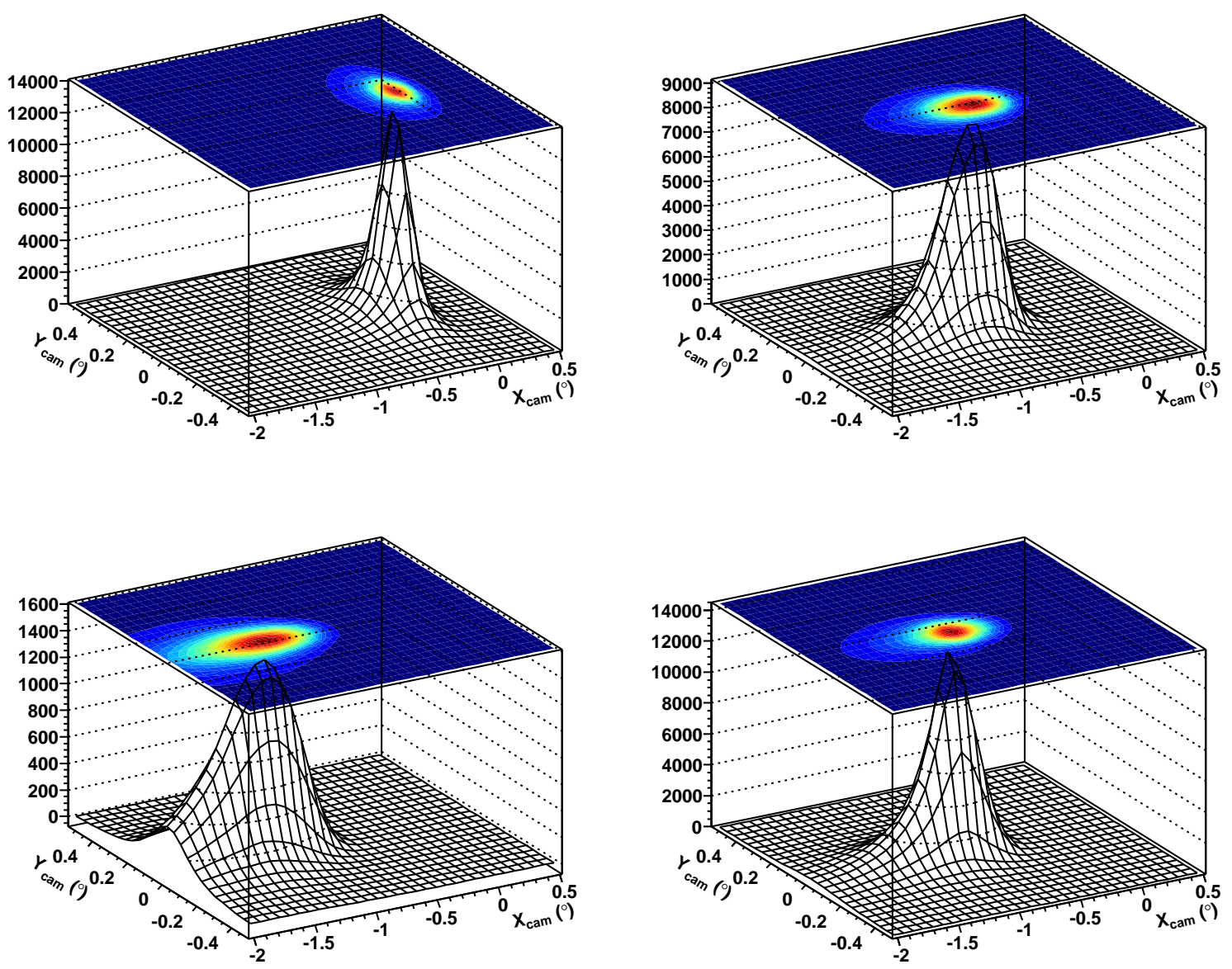

Figure 1: Image template histograms for a $1 \mathrm{TeV}$ primary gamma-ray at a core distance of $20 \mathrm{~m}$ (top left), $100 \mathrm{~m}$ (top right) and $200 \mathrm{~m}$ (bottom left) at the expected shower maximum $\left(300 \mathrm{~g} \mathrm{~cm}^{-2}\right)$. The bottom right plot shows the shower template at a core distance of $100 \mathrm{~m}$ and a $\mathrm{X}_{\max }$ of $400 \mathrm{~g} \mathrm{~cm}^{-2}$. The $z$-axis is shown in units of photoelectrons per square degree, and the $x$ - and $y$-axes are in degrees.

an event that triggers the camera were then given a weight in order to account for a number of instrumental effects and efficiencies (e.g. mirror reflectivity, quantum efficiency, integration time window), such that they represent a number of detected photoelectrons (p.e.). The average image for a given set of shower parameters was then saved in the form of a finely binned histogram, containing the expected image in a 'perfect' camera. These histograms were then oversampled with the camera pixel size, producing lookup tables for the expected image amplitude at all pixel positions within the camera (see Figure 1).

This procedure was repeated at points in a fourdimensional parameter space (described below) to produce a comprehensive library of image templates.

- 8 zenith angles $\left(0-65^{\circ}\right)$
- 2 azimuth angles

- 17 energies (0.08-100 TeV)/cos(zen)

- 25 impact distances $(0-1000 \mathrm{~m})$

Additionally events were binned in a number of bins of $\mathrm{X}_{\max }$, of width $25 \mathrm{~g} \mathrm{~cm}^{-2}$ spread around the expected $\mathrm{X}_{\max }$ for a shower of that energy. In total such a scheme should produce over 100,000 image templates, however in practice the number was somewhat smaller (less than half this total), as templates with insufficient Cherenkov photon statistics were discarded. A multidimensional linear interpolation algorithm was then used to interpolate between these templates, allowing an expected image template to be produced for any shower parameters within the above ranges.

An additional parameter ignored here is the position of the gamma-ray source within the camera field of view, which could be important due to the 


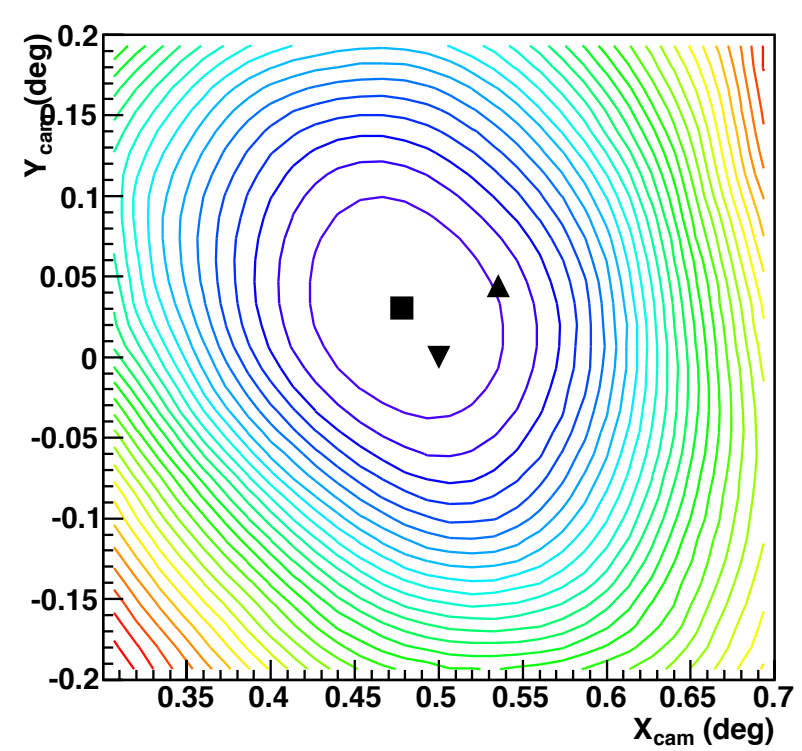

Figure 2: 2D projection of a likelihood surface for an example event in the plane of the camera. The triangle shows the reconstructed shower direction derived using a traditional Hillas-based reconstruction, the square shows the direction as reconstructed by ImPACT and the inverted triangle shows the true direction of the simulated shower.

broadening of the telescopes optical point spread function (PSF) with distance from the camera centre. However for H.E.S.S., with its Davies-Cotton optics [11], the PSF does not degrade significantly across the field of view, so events simulated on axis are sufficient in most cases.

\section{Likelihood Fitting}

Once the full set of templates for the range of possible shower parameters has been created, they must then be compared with the observed image and a fit performed to find the shower parameters that best fit the observed event.

The comparison between the expected and measured images is made using the likelihood function developed in [6]. This likelihood of a signal $s$ given an expectation of $\mu$ consists of a convolution of the Poisson distribution of each individual photoelectron $n$, with the resolution of the photosensor. Where the resolution of the photosensor is represented by a Gaussian of width $\sqrt{\sigma_{p}^{2}+n \sigma_{\gamma}^{2}}$, where $\sigma_{p}$ is the width of the pedestal (charge distribution from night sky background light and electronic noise) and $\sigma_{\gamma}$ is the width of the single photoelectron distribution $(\approx 0.5$ in H.E.S.S.).

$$
\begin{aligned}
& P\left(s \mid \mu, \sigma_{p}, \sigma_{\gamma}\right)=\sum_{n} \frac{\mu^{n} e^{-\mu}}{n ! \sqrt{2 \pi\left(\sigma_{p}^{2}+n \sigma_{\gamma}^{2}\right)}} \\
& \cdot \exp \left(-\frac{(s-n)^{2}}{2\left(\sigma_{p}^{2}+n \sigma_{\gamma}^{2}\right)}\right)
\end{aligned}
$$

The log-likelihood for that pixel is then defined such that it is distributed similarly to a $\chi^{2}$ distribution.

$$
\ln L=-2 \ln P\left(s \mid \mu, \sigma_{p}, \sigma_{\gamma}\right)
$$

In cases of large signal expectation $(\mu>5)$ the Poissonian signal fluctuations can be replaced by a Gaussian, eliminating the need to perform the sum over all photoelectrons.

$$
\begin{array}{r}
P\left(s \mid \mu \gg 0, \sigma_{p}, \sigma_{\gamma}\right) \approx \frac{1}{\sqrt{2 \pi\left(\sigma_{p}^{2}+\mu\left(1+\sigma_{\gamma}^{2}\right)\right)}} \\
\cdot \exp \left(-\frac{(s-\mu)^{2}}{2\left(\sigma_{p}^{2}+\mu\left(1+\sigma_{\gamma}^{2}\right)\right)}\right)
\end{array}
$$

More detailed derivations of equations 1 \& 3 can be found in [6].

It should be noted that when calculating the likelihood that two different gain channels are used within the H.E.S.S. camera. The high gain channel covering the range of $\sim 0-150$ p.e. and the low gain channel used above. These two channels have different levels of electronic noise and hence different pedestal widths (around 0.2 p.e. for low gain and 1 p.e. for high gain [12]) these pedestal values are broadened further during observations by the presence of night sky background light. However, in practice the contribution of the pedestal to the low gain channel (used for large signals) is small, only having an effect in pixels where the high gain channel is unavailable.

Once this per pixel likelihood function has been defined it can be simply summed over all significant pixels, selected based on the two level tail cut described in [3], with two additional rows of pixels added around the image edge, and summed over all telescopes passing selection cuts to find an event likelihood for a given set of shower parameters. This event likelihood must then be minimised in a 6-dimensional fit over direction, impact point, $X_{\max }$ and primary energy. In order to simplify the reconstruction of $X_{\max }$ it is first reconstructed using a geometrical approach, assuming depth of maximum corresponds to the brightest point in the image (calculated by taking the average position of the brightest 3 camera pixels). The minimisation can then be performed over a modification factor 
to the estimated $X_{\max }$, greatly reducing the time taken for the fit procedure.

Fitting is performed using the widely-used MINUIT [13] package, providing a fast and reliable minimisation. The algorithm finds a function minimum in the majority of cases, typically taking around 500 function calls to reach the minimum, with a computation time of $\sim 0.2-0.5$ seconds.

With such a high dimensionality, using an appropriate seed position for the fit is crucial to avoid getting trapped in a local minimum. Multiple Hillas-style reconstructions using different image cleaning values are used to generate possible fit seed positions, in addition to a the axis reconstructed using the disp method [14, 15]. Several steps are then made using a simple steepest descent algorithm from each seed position to allow the fit to approach the closest minimum and the position with the highest likelihood used to seed the MINUIT fit.

Figure 2 shows a 2 dimensional projection of the likelihood surface in the camera plane. This example event demonstrates the smoothness and well defined minimum of the likelihood surface. In this case the MIGRAD algorithm is able to find the minimum point in this function at a more accurate position than the Hillas-based reconstruction.

\subsection{Goodness of Fit}

In order to create a useful goodness of fit statistic it is necessary to know the average behaviour of the likelihood function, so that a comparison to the resultant log-likelihood of the fit can be made. The average log-likelihood for a given $\mu, \sigma_{p}$ and $\sigma_{\gamma}$ can be calculated as below:

$$
\left.\langle\ln L\rangle\right|_{\mu}=\int d s \ln L\left(s \mid \mu, \sigma_{p}, \sigma_{\gamma}\right) \times P\left(s \mid \mu, \sigma_{p}, \sigma_{\gamma}\right) .
$$

Again in the case of large signals this formula can be greatly simplified to its Gaussian limit.

$$
\left.\langle\ln L\rangle\right|_{\mu}=1+\ln (2 \pi)+\ln \left(\sigma_{p}^{2}+\mu\left(1+\sigma_{\gamma}^{2}\right)\right)
$$

More detailed derivations of equations $4 \& 5$ can be found in [6]. As equation 4 contains an integral with no analytical solution it would be expensive to compute this value on the fly, instead it is preferable to compute this value before the analysis, storing the values in lookup tables as the difference from the Gaussian expectation. This average behaviour can then be compared with the fit result to produce a goodness-of-fit statistic $(G)$.

$$
G=\frac{\sum_{i}\left[\ln L\left(s_{i} \mid \mu_{i}\right)-\left.\langle\ln L\rangle\right|_{\mu}\right]}{\sqrt{2 \times \mathrm{NdF}}}
$$

This value is constructed such that if all pixels behave like independent random variables the resultant distribution should be Gaussian in form and centred at 0 . However, figure 3 shows that this is not the case, with a long tail of high $G$ values being seen at high energies and small impact distance. This tail originates from high-energy showers at small impact distance, where visible substructure within the shower produces correlated fluctuations in multiple pixels. It should also be noted that the peak of the $G$ distribution is affected by the level of night sky background (NSB).

\section{Background Rejection}

The discrimination of the gamma-ray induced air showers from the much more numerous cosmicray induced showers is an important factor in maximising the sensitivity of an analysis. One option for background rejection is to use the aforementioned goodness-of-fit value. However, due to the strong dependence of this value of the NSB level (discussed in detail in [8] \& 6]) and the good knowledge of the single p.e. and pedestal width required, this goodness of fit value may not be stable between different observations conditions. Although this value will be a powerful background discrimination parameter, in this work it has not been used as the primary means of background rejection. However, with further work on calibration between observation conditions both this goodness of fit value and the estimated error on the direction reconstruction may be very useful in the rejection of cosmic-ray events.

Background rejection is instead performed using the boosted decision tree (BDT) based method already implemented within the H.E.S.S. framework (16] and references therein) using the root based TMVA package [17]. This method combines a series of discriminant parameters determined using a Hillas-based event reconstruction. These parameters classify events based on comparison of the image widths and lengths to the expected mean values for both gamma-rays and hadron-induced showers, the spread in energy estimates from each

\begin{tabular}{|c|c|c|c|}
\hline Config & Amp (p.e.) & $\theta^{2}\left(\mathrm{deg}^{2}\right)$ & $\zeta$ \\
\hline Hillas Std & 60 & 0.0125 & 0.84 \\
Hillas Hard & 160 & 0.01 & 0.89 \\
ImPACT Std & 60 & 0.005 & 0.83 \\
\hline
\end{tabular}

Table 1: Image selection and background rejection cuts for the three H.E.S.S. cut configurations compared in this paper. 

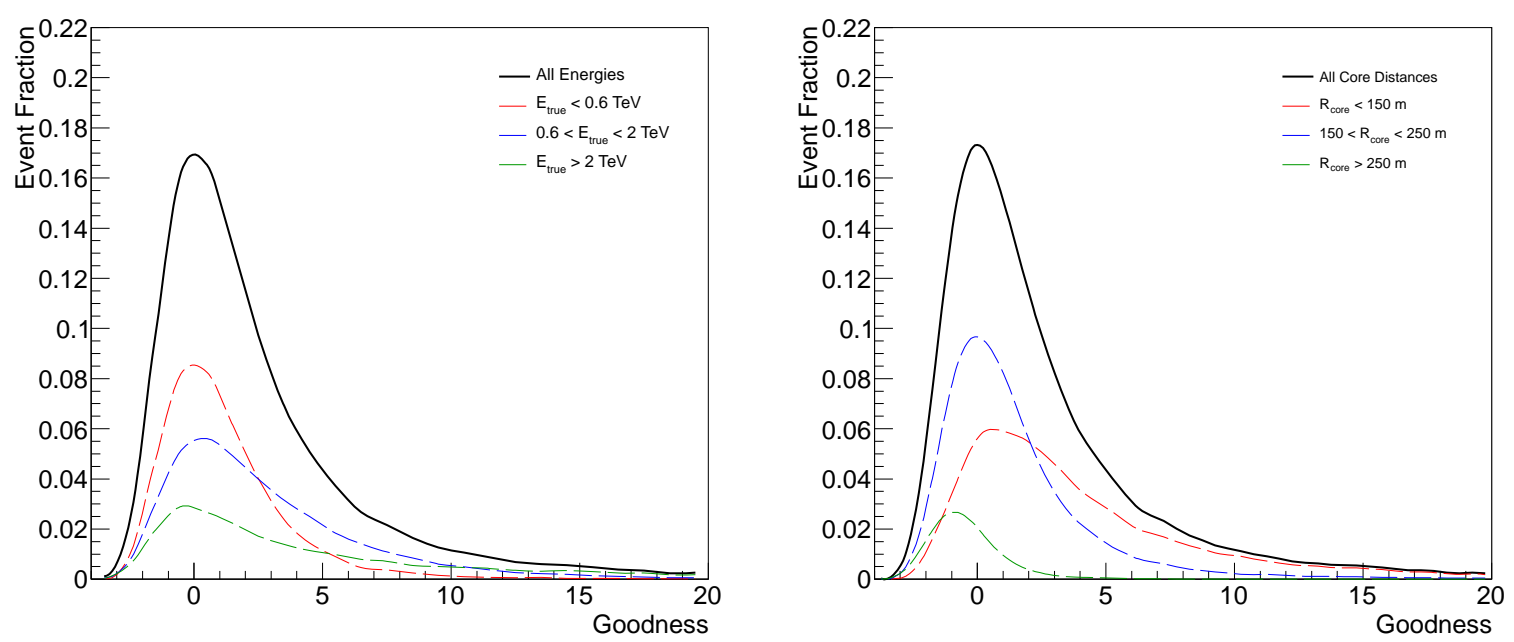

Figure 3: Distribution of goodness-of-fit for pixels with an expected signal of over 0.01 p.e. for simulated gamma-rays split into bins of true (simulated) energy (left) and bins of simulated distance of the shower core from the telescope (right). Plots are made at $20^{\circ}$ zenith angle.
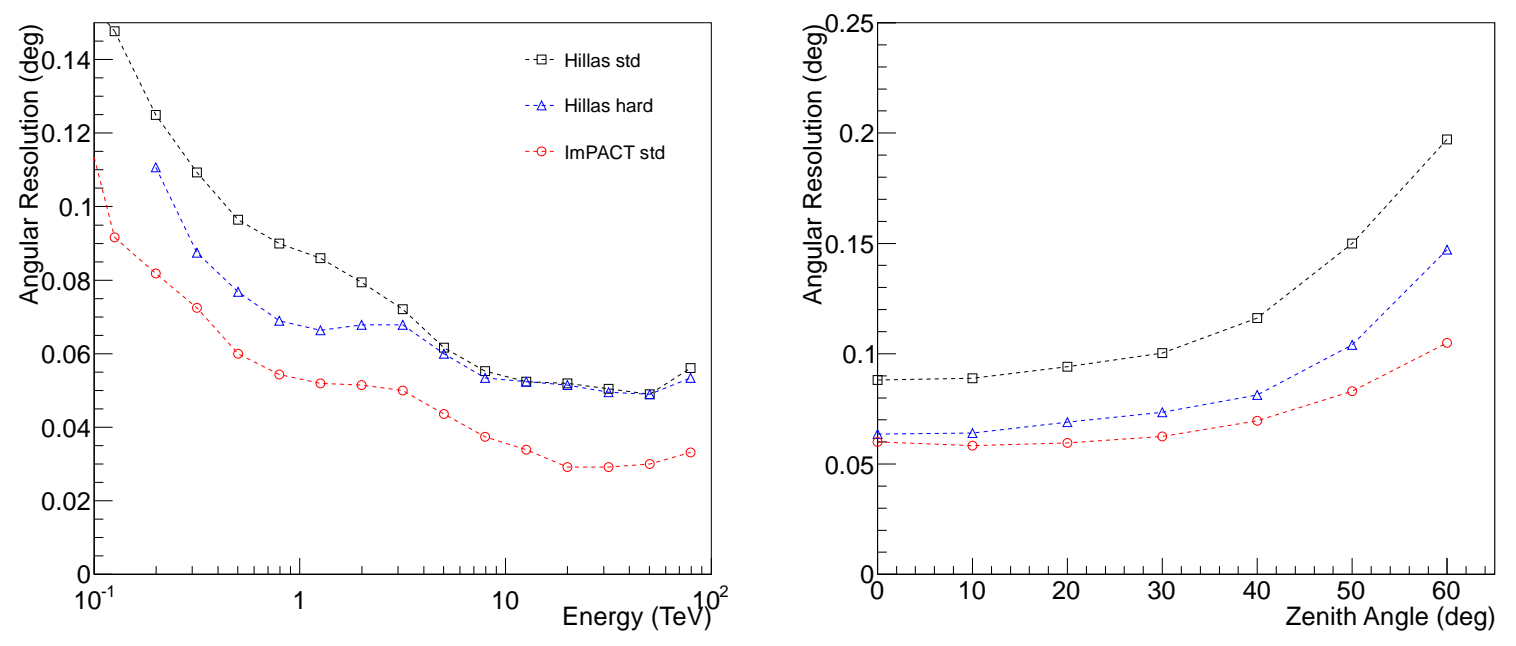

Figure 4: Angular resolution (68\% containment radius) of the ImPACT method versus simulated energy at $20^{\circ}$ zenith angle (left) and versus zenith angle (right), averaging over a spectrum of the form $d N / d E \propto$ $E^{-2}$. Results are shown in comparison to the Hillas std and hard results. 
telescope and the reconstructed height of maximum of the air shower. The distribution of the discriminant parameters obtained on gamma-ray simulations and "off"-runs (observation runs with no strong gamma-ray emission in the field of view) are then used to train a series of BDTs, created in a series of energy an zenith angle bins. Use of this multivariate analysis technique allows correlations of discriminant parameters to be effectively accounted for, producing a much more discriminating parameter $(\zeta)$ when compared to the 6 parameters independently.

Minimum amplitude (based on cleaned images), angular distance and background rejection cuts were optimised for a series of point sources with different power-law spectra. Standard cuts are optimised for a source of $10 \%$ Crab strength with a spectrum of $\mathrm{E}^{-2.6}$ and hard cuts are optimised for a source of $1 \%$ Crab strength with a spectrum of $\mathrm{E}^{-2}$. Additionally a $2^{\circ}$ cut on the position of the image centroid from the camera centre was applied, in order to remove highly truncated shower images. Table 1 shows a comparison of the cut values for the currently used Hillas TMVA cut configurations in comparison with the ImPACT standard cuts.

Currently the ImPACT reconstruction uses boosted decision trees based only on the parameters extracted from the Hillas-style reconstruction of events. However, in the future it may be possible to extract more information from the image template fit which can increase the background rejection power.

\section{Performance}

In this section the performance of the ImPACT reconstruction is compared with the performance of the standard Hillas-style reconstruction with MVA-based background rejection 16]. For brevitys sake the results of only the ImPACT method standard cuts (hereafter referred to as ImPACT $s t d)$ will be compared with both the standard and hard cuts for the Hillas-style reconstruction (hereafter referred to as Hillas std and Hillas hard).

The performance is assessed using Monte Carlo simulations of the 4 telescope H.E.S.S. IACT array, with mirrors at $70 \%$ of their nominal optical efficiency (roughly the current state of the array).

\subsection{Angular Resolution}

The angular resolution, in this case defined as the $68 \%$ containment radius of the reconstructed event positions from a point-like source, is shown as a function of energy and zenith angle in figure 4. Figure 4 (left) shows a clear improvement in angle resolution over the Hillas std across the whole energy range. This improvement ranges from around $50 \%$ at $500 \mathrm{GeV}$ where the added information has the largest effect, reducing to around $15 \%$ at $100 \mathrm{TeV}$ where a great deal of information is already provided by the Hillas parameterisation so the extra information in the fit has less impact.

An improvement is also seen in figure 4 (right) at all zenith angles, this demonstrates the weak dependence of the angular resolution on zenith angle for the ImPACT method, in contrast to the steep decline seen for the Hillas std method at high zenith angles. The rapid reduction of performance with zenith angle for the Hillas std method, is due to the apparent foreshortening in the array in the frame of the shower at large zenith. This foreshortening results in more parallel camera images in high zenith events, making event reconstruction by image axis intersection more difficult. The ImPACT method, however, considers more information than just the image axis and copes better with this problem.

The aforementioned improvement in angular resolution will help in the study of structure within large extended sources, allowing their study at smaller angular scales. Smaller angular resolution also improves the sensitivity when observing point-like sources, as a smaller integration region around the source position can be used, reducing the cosmic-ray background contamination.

\subsection{Energy Resolution}

In addition to improving in the angular resolution of the array, the ImPACT method is also able to more accurately reconstruct the energy of the primary air shower. The accuracy of reconstruction is often described by looking at the fractional deviation of the reconstructed energy from the simulated energy, figure 5 shows the mean (energy bias) and RMS (energy resolution) of this distribution as a function of energy.

The energy bias of all 3 reconstruction method is quite similar, all curves show a large bias at low energies, where event selection cuts result in only extreme examples of low energy events (with large upward fluctuations in the number of Cherenkov photons produced) being selected, which are subsequently poorly reconstructed. For the ImPACT analysis, this low energy bias is slightly less extreme than the Hillas reconstruction, resulting in a larger region of "safe" energy reconstruction, allowing spectral reconstruction to be performed at lower energies. There is almost zero bias above $500 \mathrm{GeV}$ for the ImPACT analysis, with a bias smaller than $5 \%$ being seen in this stable region for almost all possible observing conditions $\left(0-60^{\circ}\right.$ zenith angle and $0-2^{\circ}$ source offset). 

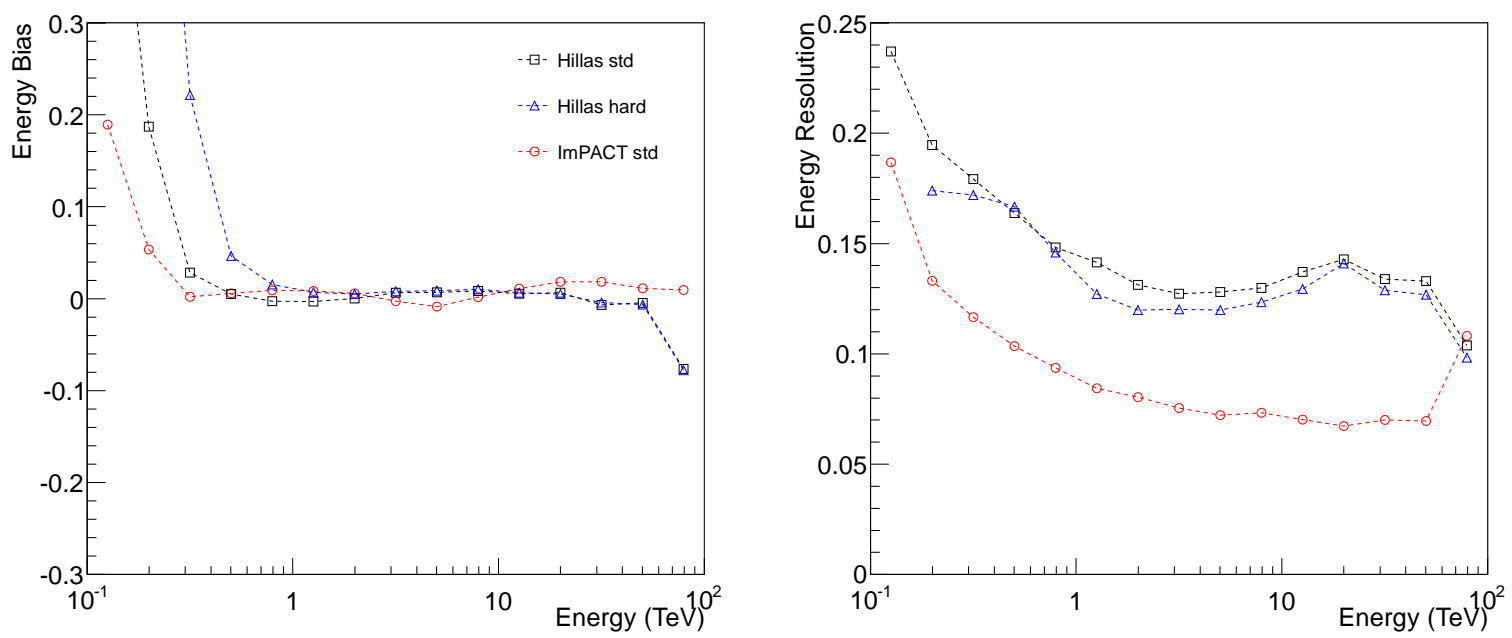

Figure 5: Fractional energy bias (left) and energy resolution (right) for the ImPACT and Hillas methods, shown as a function of simulated photon energy.
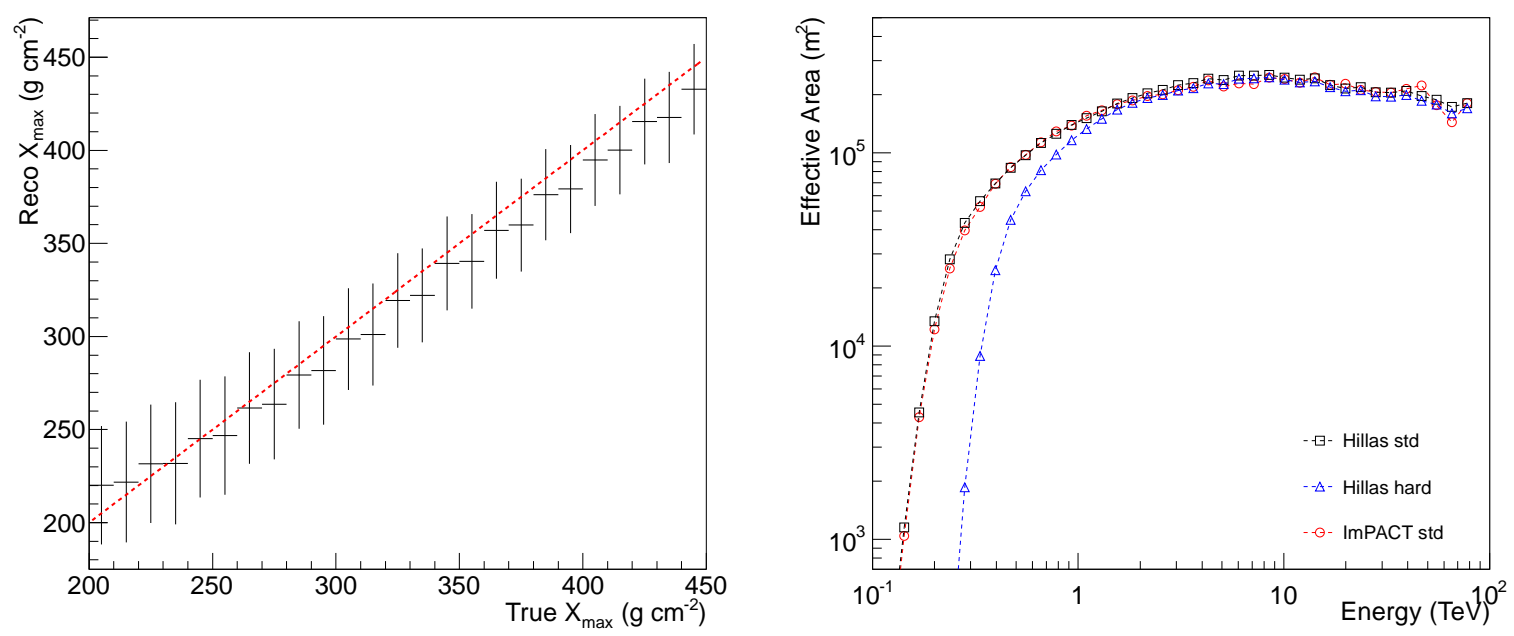

Figure 6: Left: Average reconstructed $\mathrm{X}_{\max }$ shown as a function of simulated $\mathrm{X}_{\max }$ for the ImPACT method, error bars represent the RMS of the reconstructed $\mathrm{X}_{\max }$ distribution. The dashed red line shows the line reconstructed $\mathrm{X}_{\max }=$ simulated $\mathrm{X}_{\max }$. Right: Effective collection area of the array as a function of energy for the three sets of cuts/reconstruction methods considered.

The energy resolution of the ImPACT method is significantly lower than the Hillas methods at all energies, again showing the largest improvement at low energies (around 50\% at low energies), where the additional information used in the fit is most important. Such improved energy resolution should be useful when looking for features in a source spectrum, such as the cut-off of a source at high energies, or the line emission expected from some dark matter annihilation models.

\section{3. $X_{\max }$ Resolution}

Figure 6 (left) shows a comparison of the depth of the shower maximum reconstructed from the likelihood fit with the known $\mathrm{X}_{\max }$ from the air shower simulation. The trend in the figure demonstrates that the ImPACT analysis is able to accurately reconstruct the depth of shower maximum with a small almost constant bias. In future analyses, the relatively small spread in these reconstructed values $\left(30 \mathrm{~g} \mathrm{~cm}^{-2}\right)$, may help to improve the hadronic background rejection at energies be- 
low $1 \mathrm{TeV}$.

\subsection{Effective Area}

Effective area is defined as the trigger, and subsequent event-selection, probability multiplied by the area over which simulated gamma-ray showers are scattered. For current detectors this collection area is much larger than the physical footprint of the array, due to the large extent of the Cherenkov light pool. This value is generally plotted as a function of energy, typically rising rapidly at low energies, and being relatively flat high energies, sometimes falling slowly at the highest energies as selection cuts disfavour high-energy events.

Figure 6] (right) shows the effective area after background rejection cuts for the ImPACT analysis in comparison with two Hillas cut sets. This figure shows that the effective area of the ImPACT method is very similar to that of the Hillas std method, with a deficit of less than $10 \%$ seen across the full energy range. The similarity is not surprising as the same background rejection mechanism is used for both methods and they are optimised to maximise sensitivity to the same source spectrum. The effective area of the Hillas hard method however has a significantly reduced effective area at low energies. This reduction is due to the optimisation of the cuts using a harder source spectrum, naturally leading to cuts which reject low energy events.

\subsection{Cut Sensitivity}

Figure 7 shows the improvements in point source sensitivity resulting from the increased angular resolving power of the ImPACT analysis. Shown is the time required to detect a point-like source of gamma-rays with a $\mathrm{E}^{-2.63}$ spectrum at the $5 \sigma$ level as a function of source strength.

One can see for the ImPACT method that the time required to detect a source is in many cases less than $50 \%$ of that required by the Hillas-based analysis for weak sources. For example the observation time required to detect a source of $1 \% \mathrm{Crab}$ strength is around 8.7 hours, in comparison to the 19.3 hours required for the Hillas-based analysis with multi-variate background rejection.

This large improvement in sensitivity is due to the smaller $\theta^{2}$ cut allowed by the improved PSF. For the ImPACT analysis standard cuts less than half the area of sky used in the Hillas analysis is integrated when observing a point source, resulting in a corresponding decrease in cosmic ray background events, while the effective area (and hence number of gamma-rays observed) stays roughly equal.

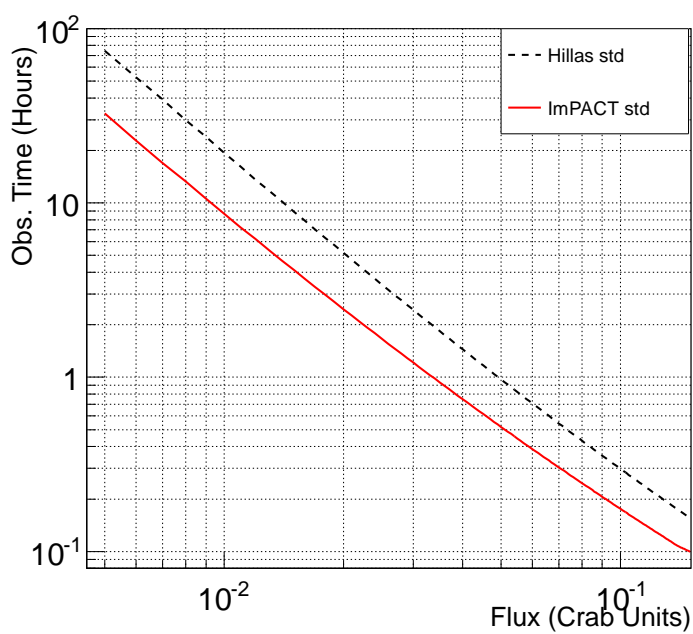

Figure 7: Plot of observation time required to detect a point-like source of a given flux (expressed as a fraction of the flux of the Crab Nebula and assuming a spectrum of the same form: $\frac{d N}{d E} \propto$ $E^{-2.63}$ ) with a statistical significance of $5 \sigma$.

\section{Source Analyses}

To demonstrate that the improvement seen in the Monte Carlo simulations translates into real world performance, the ImPACT reconstruction was tested on a strong point-like gamma-ray sources. Comparisons are then made of the source $68 \%$ containment radius against the results of the Hillas cuts.

The ideal data-set for the point spread function comparison is that taken during the strong flaring activity of the blazar PKS 2155-304 [18] in 2006. At the time, this object was the brightest source of gamma-rays yet detected by any VHE instrument and the data were taken at small zenith angles, creating a large dataset of high-quality gamma-ray events, with over 5900 events post background rejection cuts remaining in the run used.

Figure 8 show the distribution of excess events in the squared angular distance of the reconstructed event position to the test position $\left(\theta^{2}\right)$. This distribution is clearly more peaked toward zero for the ImPACT reconstruction with a significantly reduced $68 \%$ containment radius. The improvement in angular resolution seen here, combined with the tighter angular cut used is able to decrease the number of background events estimated in the source region for the ImPACT method, while keeping the estimated signal events quite similar, increasing the source significance (see table 2).

In order to test the energy reconstruction of the ImPACT reconstruction an analysis was also 


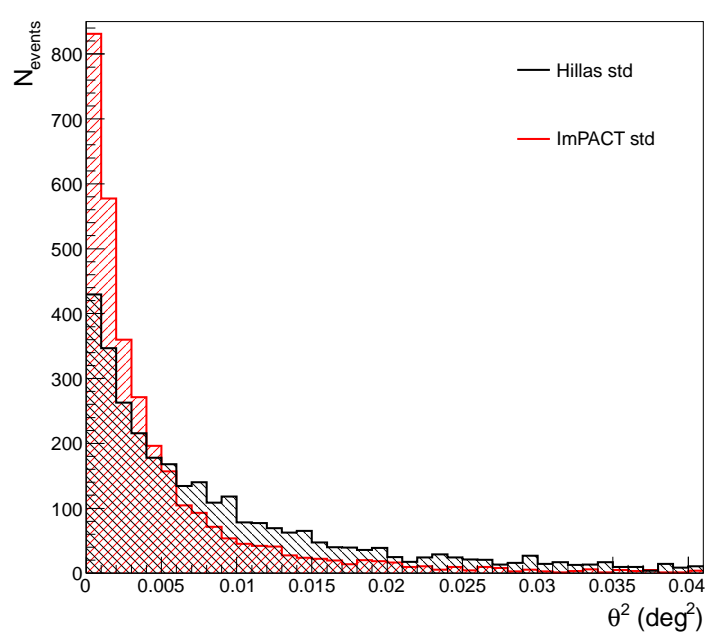

Figure 8: Distribution of squared angular distance $\left(\theta^{2}\right)$ from the source for all events passing background rejection cuts or a single run of PKS 2155-305 flare data using the Hillas std and ImPACT methods. The background level is approximately one event per bin in all bins.

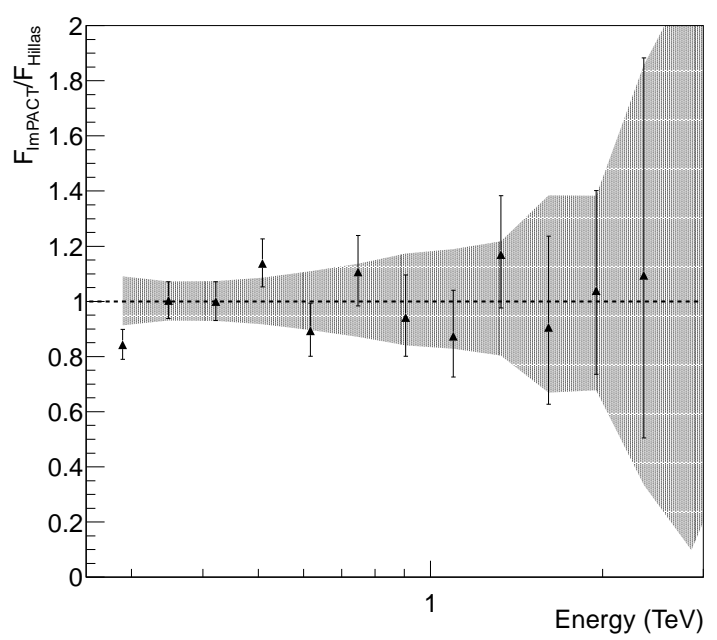

Figure 9: Ratio of flux points between ImPACT and Hillas analysis, obtained from 14 hours of H.E.S.S. PKS 2155-304 (non-flaring) data. Error bars show calculated errors on ImPACT data point, shaded area shows errors on Hillas points.

\begin{tabular}{|c|c|c|c|c|}
\hline Config & $\mathrm{N}_{\text {on }}$ & $\alpha \mathrm{N}_{\text {off }}$ & $\sigma$ & $\theta_{68}\left(^{\circ}\right)$ \\
\hline Hillas std & 2290 & 11.9 & 109 & 0.10 \\
Hillas hard & 567 & 1.4 & 58 & 0.074 \\
ImPACT std & 2279 & 4.4 & 127 & 0.067 \\
\hline
\end{tabular}

Table 2: Event statistics, significance as calculated by the method of [19] and $68 \%$ event containment radius of the first run of PKS 2155-305 flare data, for 3 H.E.S.S cut configurations.

performed on 14 hours of PKS-2155 observations (while in a non-flaring state) and an energy spectrum calculated. Figure 9 shows ratio of the ImPACT flux points with the points calculated by the Hillas method. There is very clear agreement between the two sets of flux points across the full energy range, showing the energy reconstruction performs well on H.E.S.S. data.

\section{Comparison with model analysis}

The previous section has made detailed comparisons of the ImPACT reconstruction method with a Hillas parameter based method showing large improvements in sensitivity, however some comparison to the similar model analysis must also be made. However, it must be noted that the simulations used in the ImPACT results and those in the model results were produced with different simulation chains and there may be some systematic differences between the results of the two chains (typically at the $5 \%$ level in total Cherenkov yield [20]).

Figure 10 shows a comparison between the angular resolution of the ImPACT analysis and the model analysis for standard cuts in both analyses. It can be seen in this plot that at low energies the angular resolution of the ImPACT reconstruction is similar to that of the model analysis. This similarity is expected due to the good description of the air shower by the semi-analytical model at low energies and the relative similarity of the fit procedure. However, at higher energies the angular resolution of the ImPACT reconstruction is somewhat improved over that of model, due to difficultly of reproducing the behaviour of air showers with the semi-analytical model. This improvement can also be seen in the post-cuts effective area at high energies, with the rapid drop-off in effective area demonstrating the difficultly of the model analysis to reconstruct events at high energies, where no such problem exists for ImPACT. Above $10 \mathrm{TeV}$ H.E.S.S. observations are typically in the statistics limited regime, such an improvement in high energy effective area should linearly translate into an 

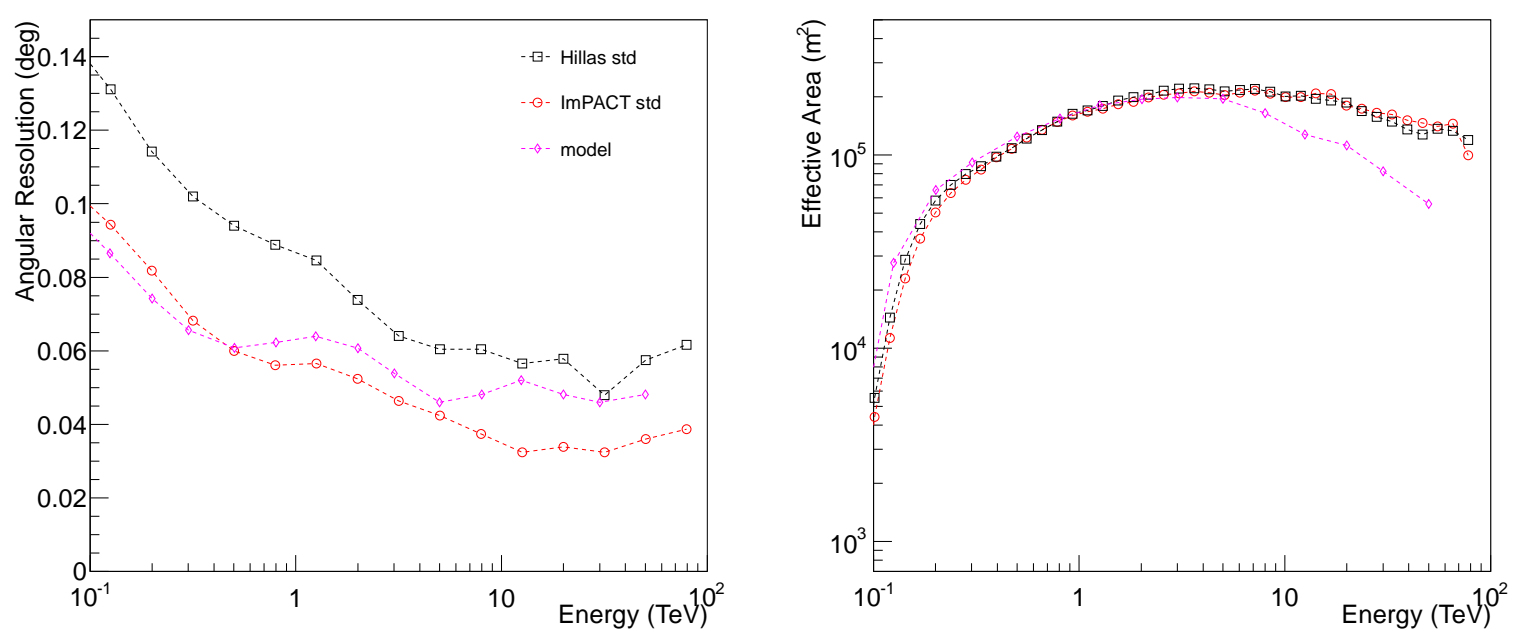

Figure 10: Comparison of key performance results from the ImPACT event reconstruction with the model reconstruction (data points reproduced from [6]). Left: Angular resolution (68\% containment radius) as a function of simulated energy. Right: Post cuts effective area as a function of energy. All curves are calculated at zenith, using standard cuts in all analyses, and HESS simulations with 100\% of the nominal optical efficiency.

improvement in sensitivity.

\section{Conclusions}

We have demonstrated a high performance likelihood-based reconstruction method for the H.E.S.S. telescope, based on expected image templates generated from Monte Carlo air shower simulations. This technique has been proven to be extremely successful, reconstructing events with significantly more accuracy than the standard reconstruction algorithm. Some improvement has also been demonstrated over the model reconstruction, especially at the highest energies where semianalytical modelling of the air shower becomes difficult. Use of the ImPACT reconstruction is able to provide an sensitivity improvement of around a factor of 2 in observation time over traditional Hillas-based reconstruction for point source observations. Additionally the improvement in angular resolution offers more detailed imaging of extended sources.

Although some performance improvements have been shown over the existing, semi-analytical model based approach to template generation, the major advantage of production via MC simulation is the robustness of this approach against changes in telescope hardware. For example the H.E.S.S. observatory has recently commissioned a fifth $26 \mathrm{~m}$ diameter telescope (CT5) at the centre of the array, which requires the production of a new set of image templates. In order to generate these new templates one can simply re-run the template generation step using the CT5 simulation configuration, whereas using a model based approach the model must be changed to accurately reflect the new hardware. This robustness will be especially important for the next generation of Cherenkov telescopes, such as the Cherenkov Telescope Array, which plan to use multiple telescope types within the same array.

Additionally, this template generation mechanism can in principle be implemented to allow reconstruction of any particle type. In the case of protons the large shower to shower fluctuations present may make this difficult, however for other particles such as electrons or Iron nuclei it may be possible to reconstruct and separate events using this method.

\section{Acknowledgements}

We thank Prof. C Stegmann, spokesperson for the H.E.S.S. Collaboration and Prof G. Fontaine, chairperson of the Collaboration board, for allowing us to use data from the H.E.S.S. array in this publication. We would like to thank Dr. V. Marandon and Dr. K. Bernlöhr for technical assistance in the implementation of the analysis. Finally we would like to thank the H.E.S.S. collaboration for useful discussion of the analysis procedure.

[1] J. A. Hinton. The status of the HESS project. New Astronomy Reviews, 48:331-337, April 2004. 
[2] A. M. Hillas. Cerenkov light images of EAS produced by primary gamma. In International Cosmic Ray Conference, volume 3 of International Cosmic Ray Conference, pages 445-448, August 1985.

[3] F. Aharonian, A. G. Akhperjanian, A. R. BazerBachi, M. Beilicke, W. Benbow, D. Berge, K. Bernlöhr, C. Boisson, O. Bolz, V. Borrel, I. Braun, F. Breitling, A. M. Brown, R. Bühler, I. Büsching, S. Carrigan, P. M. Chadwick, L.-M. Chounet, R. Cornils, L. Costamante, B. Degrange, H. J. Dickinson, A. DjannatiAtaï, L. O'C. Drury, G. Dubus, K. Egberts, D. Emmanoulopoulos, P. Espigat, F. Feinstein, E. Ferrero, A. Fiasson, G. Fontaine, S. Funk, S. Funk, Y. A. Gallant, B. Giebels, J. F. Glicenstein, P. Goret, C. Hadjichristidis, D. Hauser, M. Hauser, G. Heinzelmann, G. Henri, G. Hermann, J. A. Hinton, W. Hofmann, M. Holleran, D. Horns, A. Jacholkowska, O. C. de Jager, B. Khélifi, N. Komin, A. Konopelko, K. Kosack, I. J. Latham, R. Le Gallou, A. Lemière, M. LemoineGoumard, T. Lohse, J. M. Martin, O. MartineauHuynh, A. Marcowith, C. Masterson, T. J. L. McComb, M. de Naurois, D. Nedbal, S. J. Nolan, A. Noutsos, K. J. Orford, J. L. Osborne, M. Ouchrif, M. Panter, G. Pelletier, S. Pita, G. Pühlhofer, M. Punch, B. C. Raubenheimer, M. Raue, S. M. Rayner, A. Reimer, O. Reimer, J. Ripken, L. Rob, L. Rolland, G. Rowell, V. Sahakian, L. Saugé, S. Schlenker, R. Schlickeiser, U. Schwanke, H. Sol, D. Spangler, F. Spanier, R. Steenkamp, C. Stegmann, G. Superina, J.-P. Tavernet, R. Terrier, C. G. Théoret, M. Tluczykont, C. van Eldik, G. Vasileiadis, C. Venter, P. Vincent, H. J. Völk, S. J. Wagner, and M. Ward. Observations of the Crab nebula with HESS. Astronomy \& Astrophysics, 457:899-915, October 2006.

[4] A. Barrau, R. Bazer-Bachi, E. Beyer, H. Cabot, M. Cerutti, L. M. Chounet, G. Debiais, B. Degrange, H. Delchini, J. P. Denance, G. Descotes, J. P. Dezalay, A. Djannati-Atai, D. Dumora, P. Espigat, B. Fabre, P. Fleury, G. Fontaine, R. George, C. Ghesquiere, J. Gilly, P. Goret, C. Gouiffes, J. C. Gouillaud, C. Gregory, I. A. Grenier, L. Iacoucci, L. Kalt, S. Le Bohec, I. Malet, C. Meynadier, J. P. Mols, P. M. de Freitas, R. Morano, G. Morinaud, F. Munz, M. Palatka, T. A. Palfrey, E. Pare, Y. Pons, M. Punch, J. Quebert, K. Ragan, C. Renault, M. Rivoal, L. Rob, P. Schovanek, D. Smith, A. Tabary, J. P. Tavernet, F. Toussenel, and J. Vrana. The CAT imaging telescope for very-high-energy gamma-ray astronomy. $\mathrm{Nu}$ clear Instruments and Methods in Physics Research A, 416:278-292, October 1998.

[5] S. Le Bohec, B. Degrange, M. Punch, A. Barrau, R. Bazer-Bachi, H. Cabot, L. M. Chounet, G. Debiais, J. P. Dezalay, A. Djannati-Atai, D. Dumora, P. Espigat, B. Fabre, P. Fleury, G. Fontaine, R. George, C. Ghesquiere, P. Goret, C. Gouiffes, I. A. Grenier, L. Iacoucci, I. Malet, C. Meynadier, F. Munz, T. A. Palfrey, E. Pare, Y. Pons, J. Quebert, K. Ragan, C. Renault, M. Rivoal, L. Rob, P. Schovanek, D. Smith, J. P. Tavernet, and J. Vrana. A new analysis method for very high definition imaging atmospheric Cherenkov telescopes as applied to the CAT telescope. $\mathrm{Nu}$ clear Instruments and Methods in Physics Research A, 416:425-437, October 1998.

[6] M. de Naurois and L. Rolland. A high performance likelihood reconstruction of $\gamma$-rays for imaging atmospheric Cherenkov telescopes. Astroparticle Physics, 32:231-252, December 2009.

[7] M. Lemoine-Goumard, B. Degrange, and M. Tluczykont. Selection and 3D-reconstruction of gamma-ray-induced air showers with a stereoscopic system of atmospheric Cherenkov telescopes.
Astroparticle Physics, 25:195-211, April 2006.

[8] Y. Becherini, A. Djannati-Ataï, V. Marandon, M. Punch, and S. Pita. A new analysis strategy for detection of faint $\gamma$-ray sources with Imaging Atmospheric Cherenkov Telescopes. Astroparticle Physics, 34:858-870, July 2011.

[9] D. Heck, J. Knapp, J. N. Capdevielle, G. Schatz, and T. Thouw. CORSIKA: a Monte Carlo code to simulate extensive air showers. February 1998.

[10] K. Bernlöhr. Simulation of imaging atmospheric Cherenkov telescopes with CORSIKA and sim_telarray. Astroparticle Physics, 30:149-158, October 2008.

[11] J. M. Davies and I. S. Cotton. Design of the Quartermaster Solar Furnace. Solar Energy Sci. Eng., 1:16-22, 1957.

[12] F. Aharonian, A. G. Akhperjanian, K.-M. Aye, A. R. Bazer-Bachi, M. Beilicke, W. Benbow, D. Berge, P. Berghaus, K. Bernlöhr, O. Bolz, C. Boisson, C. Borgmeier, F. Breitling, A. M. Brown, P. M. Chadwick, V. R. Chitnis, L.-M. Chounet, R. Cornils, L. Costamante, B. Degrange, O. C. de Jager, A. Djannati-Ataï, L. O. '. Drury, T. Ergin, P. Espigat, F. Feinstein, P. Fleury, G. Fontaine, S. Funk, Y. A. Gallant, B. Giebels, S. Gillessen, P. Goret, J. Guy, C. Hadjichristidis, M. Hauser, G. Heinzelmann, G. Henri, G. Hermann, J. Hinton, W. Hofmann, M. Holleran, D. Horns, I. Jung, B. Khélifi, N. Komin, A. Konopelko, I. J. Latham, R. L. Gallou, M. Lemoine, A. Lemière, N. Leroy, T. Lohse, A. Marcowith, C. Masterson, T. J. L. McComb, M. de Naurois, S. J. Nolan, A. Noutsos, K. J. Orford, J. L. Osborne, M. Ouchrif, M. Panter, G. Pelletier, S. Pita, M. Pohl, G. Pühlhofer, M. Punch, B. C. Raubenheimer, M. Raue, J. Raux, S. M. Rayner, I. Redondo, A. Reimer, O. Reimer, J. Ripken, M. Rivoal, L. Rob, L. Rolland, G. Rowell, V. Sahakian, L. Sauge, S. Schlenker, R. Schlickeiser, C. Schuster, U. Schwanke, M. Siewert, H. Sol, R. Steenkamp, C. Stegmann, J.-P. Tavernet, C. G. Théoret, M. Tluczykont, D. J. van der Walt, G. Vasileiadis, P. Vincent, B. Visser, H. J. Volk, and S. J. Wagner. Calibration of cameras of the H.E.S.S. detector. Astroparticle Physics, 22:109-125, November 2004.

[13] F. James and M. Roos. Minuit: A System for Function Minimization and Analysis of the Parameter Errors and Correlations. Comput.Phys.Commun., 10:343$367,1975$.

[14] W. Hofmann, I. Jung, A. Konopelko, H. Krawczynski, H. Lampeitl, and G. Pühlhofer. Comparison of techniques to reconstruct VHE gamma-ray showers from multiple stereoscopic Cherenkov images. Astroparticle Physics, 12:135-143, November 1999.

[15] C.-C. Lu and for the H. E. S. S. Collaboration. Improving the H.E.S.S. angular resolution using the Disp method. ArXiv e-prints, October 2013.

[16] S. Ohm, C. van Eldik, and K. Egberts. $\gamma /$ hadron separation in very-high-energy $\gamma$-ray astronomy using a multivariate analysis method. Astroparticle Physics, 31:383-391, June 2009.

[17] A. Hoecker, P. Speckmayer, J. Stelzer, J. Therhaag, E. von Toerne, H. Voss, M. Backes, T. Carli, O. Cohen, A. Christov, D. Dannheim, K. Danielowski, S. HenrotVersille, M. Jachowski, K. Kraszewski, A. Krasznahorkay, Jr., M. Kruk, Y. Mahalalel, R. Ospanov, X. Prudent, A. Robert, D. Schouten, F. Tegenfeldt, A. Voigt, K. Voss, M. Wolter, and A. Zemla. TMVA Toolkit for Multivariate Data Analysis. ArXiv Physics e-prints, March 2007.

[18] F. Aharonian, A. G. Akhperjanian, A. R. BazerBachi, B. Behera, M. Beilicke, W. Benbow, D. Berge, 
K. Bernlöhr, C. Boisson, O. Bolz, V. Borrel, T. Boutelier, I. Braun, E. Brion, A. M. Brown, R. Bühler, I. Büsching, T. Bulik, S. Carrigan, P. M. Chadwick, A. C. Clapson, L.-M. Chounet, G. Coignet, R. Cornils, L. Costamante, B. Degrange, H. J. Dickinson, A. Djannati-Ataï, W. Domainko, L. O. Drury, G. Dubus, J. Dyks, K. Egberts, D. Emmanoulopoulos, P. Espigat, C. Farnier, F. Feinstein, A. Fiasson, A. Förster, G. Fontaine, S. Funk, S. Funk, M. Füßling, Y. A. Gallant, B. Giebels, J. F. Glicenstein, B. Glück, P. Goret, C. Hadjichristidis, D. Hauser, M. Hauser, G. Heinzelmann, G. Henri, G. Hermann, J. A. Hinton, A. Hoffmann, W. Hofmann, M. Holleran, S. Hoppe, D. Horns, A. Jacholkowska, O. C. de Jager, E. Kendziorra, M. Kerschhaggl, B. Khélifi, N. Komin, K. Kosack, G. Lamanna, I. J. Latham, R. Le Gallou, A. Lemière, M. Lemoine-Goumard, J.-P. Lenain, T. Lohse, J. M. Martin, O. Martineau-Huynh, A. Marcowith, C. Masterson, G. Maurin, T. J. L. McComb, R. Moderski, E. Moulin, M. de Naurois, D. Nedbal, S. J. Nolan, J.-P. Olive, K. J. Orford, J. L. Osborne, M. Ostrowski, M. Panter, G. Pedaletti, G. Pelletier, P.O. Petrucci, S. Pita, G. Pühlhofer, M. Punch, S. Ranchon, B. C. Raubenheimer, M. Raue, S. M. Rayner, M. Renaud, J. Ripken, L. Rob, L. Rolland, S. RosierLees, G. Rowell, B. Rudak, J. Ruppel, V. Sahakian, A. Santangelo, L. Saugé, S. Schlenker, R. Schlickeiser, R. Schröder, U. Schwanke, S. Schwarzburg, S. Schwemmer, A. Shalchi, H. Sol, D. Spangler, E. Stawarz, R. Steenkamp, C. Stegmann, G. Superina, P. H. Tam, J.-P. Tavernet, R. Terrier, C. van Eldik, G. Vasileiadis, C. Venter, J. P. Vialle, P. Vincent, M. Vivier, H. J. Völk, F. Volpe, S. J. Wagner, M. Ward, and A. A. Zdziarski. An Exceptional Very High Energy GammaRay Flare of PKS 2155-304. Astrophysical Journal Letters, 664:L71-L74, August 2007.

[19] T.-P. Li and Y.-Q. Ma. Analysis methods for results in gamma-ray astronomy. The Astrophysical Journal, 272:317-324, September 1983.

[20] K. Bernlöhr, A. Barnacka, Y. Becherini, O. Blanch Bigas, E. Carmona, P. Colin, G. Decerprit, F. Di Pierro, F. Dubois, C. Farnier, S. Funk, G. Hermann, J. A. Hinton, T. B. Humensky, B. Khélifi, T. Kihm, N. Komin, J.-P. Lenain, G. Maier, D. Mazin, M. C. Medina, A. Moralejo, S. J. Nolan, S. Ohm, E. de Oña Wilhelmi, R. D. Parsons, M. Paz Arribas, G. Pedaletti, S. Pita, H. Prokoph, C. B. Rulten, U. Schwanke, M. Shayduk, V. Stamatescu, P. Vallania, S. Vorobiov, R. Wischnewski, T. Yoshikoshi, A. Zech, and CTA Consortium. Monte Carlo design studies for the Cherenkov Telescope Array. Astroparticle Physics, 43:171-188, March 2013. 